\title{
Analysis of nonlocal model of compressible fluid in $1-\mathrm{D}$
}

\author{
Ewelina Zatorska \\ March 7, 2018
Institute of Applied Mathematics and Mechanics
University of Warsaw
ul.Banacha 2, 02-097 Warszawa, Poland
E-MAIL: E.KAMINSKA@MIMUW.EDU.PL

\begin{abstract}
We study a nonlocal modification of the compressible Navier-Stokes equations in mono dimensional case with a boundary condition characteristic for the free boundaries problem. From the formal point of view our system is an intermediate between the Euler and Navier-Stokes equations. Under certain assumptions, imposed on initial data and viscosity coefficient, we obtain the local and global existence of solutions. Particularly, we show the uniform in time bound on the density of fluid.
\end{abstract}

Keywords: Navier-Stokes equations, barotropic compressible viscous fluid, weak solution, global existence, nonlocal models

Mathematics Subject Classification: 76N10; 35Q30

\section{Introduction}

We investigate one-dimensional model of motion for a barotropic compressible fluid. The model is described in the Lagrangian mass coordinates $(x, t)$ by the system of equations

$$
\begin{aligned}
v_{t}+p\left(\xi^{-1}\right)_{x} & =\mu(\mathbf{T} v)_{x x}, \\
\xi_{t}-v_{x} & =0
\end{aligned}
$$

where $v$-the velocity and $\xi$-the specific volume $\left(\xi^{-1}(x, t)=\varrho(x, t)\right.$ - the density) are unknown functions; $\mu$ is a positive constant viscosity coefficient and $p=p(\varrho)=p\left(\xi^{-1}\right)$ describes the pressure of fluid as a function of density.

By conservation of total mass and after normalising the total initial mass to 1 the free boundaries become fixed $(x \in[0,1])$ in terms of Lagrangian coordinates [1] and our system may by supplied with the Neumann boundary condition given as follows

$$
\mu(\mathbf{T} v)_{x}-\left.p\left(\xi^{-1}\right)\right|_{x=0}=\mu(\mathbf{T} v)_{x}-\left.p\left(\xi^{-1}\right)\right|_{x=1}=-P
$$


where $P$ stands for the constant external pressure.

We assume constitutive equation as for isentropic processes

$$
p(\varrho)=a \varrho^{\gamma} \quad \text { with constants } \gamma>3 \text { and } a>0,
$$

additionally we introduce a function $G(\varrho)=\frac{a}{\gamma-1} \varrho^{\gamma-1}$.

The initial conditions are $v(x, 0)=v_{0}(x), \xi(x, 0)=\xi_{0}(x)$, and they satisfy

$$
\int_{0}^{1} v_{0}(x) d x=0, \quad 0<\xi_{0-} \leq \xi_{0}(x) \leq \xi_{0+}<\infty, \quad \xi_{0}(0)=\xi_{0}(1),
$$

for some positive constants $\xi_{0-}, \xi_{0+}$.

From the physical point of view the assumption $\gamma>3$ is not satisfactory, since for most gases the heat capacity ratio is about $\frac{7}{5}$. On the other hand, it allows for simplification while we show the upper bound on a density for approximative system. This is just a technical assumption often used for the sake of simplicity and transparency of the proof [11] and there are some well known techniques extending the results for $\gamma>1$ for the similar type of equations [7], [9]. It seems that for our model the case $1<\gamma \leq 3$ can also be studied, as far as strong convergence and local existence results are concerned, and we shall come back to this problem in a future publication.

On the r.h.s. of first equation of system (1) we find a pseudo-differential operator acting on the velocity function $v$, being a modification of the standard Laplacian. Its definition is based on the properties of space of weak solutions to $(1)+(2)$ which is the Neumann-boundary problem. We immerse a space of weak solutions in $L_{2}(0,1)$ - the closure of linear combinations of smooth functions that form a standard base for the Neumann-boundary problem:

$$
w_{k}(x)=\frac{\cos (\pi k x)}{\|\cos (\pi k \cdot)\|_{L_{2}(0,1)}} \quad k=0,1, \ldots
$$

Thus, we may describe any function $f \in L_{2}(0,1)$ as $f(x)=\sum_{k=0}^{\infty} f_{k} w_{k}(x)$. Staying within above notation we define an operator

$$
\mathbf{T}: L_{2}(0,1) \rightarrow L_{2}(0,1), \quad \mathbf{T} f(x)=\sum_{k=R+1}^{\infty} f_{k} w_{k}(x),
$$

where we assume that $R$ is a positive fixed natural number.

The operator $\mathbf{T}$ is thus a projector which omits first $R$ modes of the function. This feature causes that the dissipation of energy appears (see Lemma 4) only for high fluctuations and does not involve low modes (lower than $R$ ).

If the system exhibits only low modes, the right hand side of the first equation in (1) disappears, thus the equations have features of the Euler's system for compressible inviscid flow; for modes grater than $R$ we have Navier-Stokes type equations with the dissipation of energy proportional to a degenerate viscosity coefficient $\mu>0$.

For one-dimensional Navier-Stokes system with external force it has been proved (see 
$[14],[10])$ that the global solutions exist, and that any solution tends to the stationary solution [8]. Similar results exist for system with density-dependent viscosity [3]. There are also some attempts developing this theory for descriptions of heat-conducting gases undergoing dynamic combustion [6] or/and including radiative and self-gravitation effects [4]. These models arise from astrophysical hydrodynamics and are usable to describe how the matter behaves inside a viscous gaseous star [13], [5]. Our system may be treated as a simple mathematical model of a single layer of star's atmosphere bounded by an external pressure $P$, if the star is spherically symmetrical and the radius is suitable large to justify passing over the influence of curvature.

The objective of this paper is to show an existence of regular solutions to the problem $(1)+(2)$. To obtain a global in time existence we need first a local in time existence and then some information about solutions uniformly in time. Local existence for the Navier-Stokes equations in a general three-space dimensional case has been recognized alike for the homogenous Dirichlet problem [7] as well as for the Neumann condition given on a free boundary [12]. Nevertheless, the method presented here is an application of other technique, introduced by Kazhikhov (see [14]), after noticing that on the level of the Galerkin approximations the first equation of (1) may be stated as follows

$$
v_{t}+p\left(\xi^{-1}\right)_{x}=\mu v_{x x}-\mu((1-\mathbf{T}) v)_{x x}
$$

where $(1-\mathbf{T}) v_{x x}$ is an analytic function, since $R$ is a fixed positive number, and its norm is controlled by the norm of $v$ due to the energy equality, thus it may be treated as given one as an external force $f$. This approach causes that all the constants that appear throughout the paper are highly dependent on $R$, but we are not interested in knowing the precise expression for this dependence as we assumed $R$ to be a constant, positive parameter of the system.

To find the regular solutions to the problem (1), we should validate its well posedness in the classical sense. To avoid such difficulties we will work with weak solutions to $(1)+(2)$, although the final regularity allows us to call them regular.

Definition (Weak solutions). We say a pair of functions

$$
v \in W_{2}^{2,1}((0,1) \times(0, T)) \quad \text { and } \quad \xi \in L_{\infty}\left(0, T ; H^{1}(0,1)\right)
$$

is a weak solution to the problem (1)+(2) provided:

1. for each $\varphi \in C^{1}\left(0, T ; H^{1}(0,1)\right), \int_{0}^{1} \varphi(x, t) d x=0$, the equalities

$$
\begin{gathered}
\left(v_{t}, \varphi\right)-\left(p\left(\xi^{-1}\right)-P, \varphi_{x}\right)+\mu\left(\mathbf{T} v_{x}, \varphi_{x}\right)=0 \\
\xi_{t}-v_{x}=0
\end{gathered}
$$

are fulfilled in the sense of distributions on time interval [0,T] and 2. $v(x, 0)=v_{0}, \xi(x, 0)=\xi_{0}$.

This definition, as the only in this paper, will be simply referred to as "the Definition" in the further part, and the system (6) as "the weak formulation".

We require from $v$ regularity, which is not optimal to the weak formulation, however 
such high smoothness will be necessary to show uniqueness of the solution defined above.

The local existence of solutions is provided due to theorem

Theorem A (Local in time existence). Assuming (3), (4), $R$ is a fixed positive number and that $v_{0}, \xi_{0} \in H^{1}(0,1)$, there exists $T_{0}>0$ such that there exists unique solution to the problem (1)+(2) in the sense of Definition on time interval [0,T], $T<T_{0}$. Moreover, $\xi$ is strictly positive on time interval $[0, T]$.

The proof is based on the Galerkin approximations and an additional energy estimate that enables to control the norm of nonlinear term.

Our principal result reads as follows

Theorem B (Global in time existence). Assuming (3), (4), $\mu \ll 1, R$ is a fixed positive number and that $v_{0}, \xi_{0} \in H^{1}(0,1)$, there exists global in time solution to problem (1)+(2) such that

$$
\begin{gathered}
v \in W_{2(l o c)}^{2,1}((0,1) \times(0, \infty)), \quad \xi \in L_{\infty}\left(0, \infty ; H^{1}(0,1)\right), \\
\text { and } 0<\xi_{-} \leq \xi(x, t) \leq \xi_{+}<\infty,
\end{gathered}
$$

for all $(x, t) \in(0,1) \times[0, \infty)$, where $\xi_{-}, \xi_{+}$are strictly positive constants.

The main difficulty in proof of Theorem B is to show uniformly boundedness of the density $\varrho(x, t) \geq \xi_{-}$. The idea comes from P. B. Mucha [2] and requires an assumption of smallness of viscosity coefficient $\mu$, which is the most interesting case from the physical point of view. For a sake of Neumann-boundary condition we have a global existence without assumption of smallness of initial data. In case of Dirichlet boundary condition, smallness of data is required, but it depends only on $\gamma[10]$.

In the following section we investigate behaviour of solution to (1) on the boundary in classical sense, to introduce a suitable form of approximate solutions in the Galerkin method.

Section 3 is devoted to the proof of Theorem A. We start with energy equality and estimate, from which we obtain a sufficient information to pass to weak limit and justify the local existence of solutions. However, to show uniqueness, there is a need to improve regularity of $v$.

In Section 4, we present the proof of Theorem B. Global in time estimates for $v, G\left(\xi^{-1}\right)$ and the upper bound on $\xi$ enable to justify that $\xi$ belongs to $L_{\infty}\left(0, \infty ; H^{1}(0,1)\right)$ and, as a consequence, separation of $\xi$ from zero, globally in time.

Notations:

$$
\begin{gathered}
\frac{\partial f}{\partial a}=\partial_{a} f=f_{a}, \quad(f, g)=\int_{0}^{1} f \cdot g d x \\
\|f\|_{W_{2}^{2,1}((0,1) \times(0, T))}=\sum_{k=0}^{2}\left\|\partial_{x}^{k} f\right\|_{L_{2}((0,1) \times(0, T))}+\sum_{k=0}^{1}\left\|\partial_{t}^{k} f\right\|_{L_{2}((0,1) \times(0, T))} .
\end{gathered}
$$




\section{An estimate of the solution on the boundary}

The first equation of (1) together with boundary and initial conditions lead to a conclusion that $\int_{0}^{1} v(x, t) d x=0$, thus it is reasonable to look for function $v(x, t)$ of the structure

$$
v(x, t)=\sum_{k=1}^{\infty} \lambda_{k}(t) \omega_{k}(x),
$$

where the $\lambda_{k}(t)$ are at least of class $C^{1}(0, \infty)$.

Lemma 1. Assume (4). Then for all $t \geq 0$ there exists a $C^{1}(0, t)$ function $\xi^{*}(t)$ being a unique solution to (2), i.e.

$$
\xi(0, t)=\xi(1, t)=\xi^{*}(t)
$$

Moreover

$$
0<\bar{\xi}_{-} \leq \xi^{*}(t) \leq \bar{\xi}_{+}<\infty
$$

where $\bar{\xi}_{-}$and $\bar{\xi}_{+}$are independent of $t$.

Proof. According to definition of the operator $\mathbf{T}$ and to the structure of function $v$ we get $\left.((1-\mathbf{T}) v)_{x}\right|_{(b, t)}=0$, for $b \in\{0,1\}$. Therefore, one can reduce (2) to an ODE

$$
\mu \xi_{t}=\frac{a}{\xi^{\gamma}}-P
$$

subject to the initial condition $\xi(x, 0)=\xi_{0}(x)$. From the classical theory, there exists a unique solution with continuous first time derivative that may be extended to the whole half line.

Since $\xi_{0}(0)=\xi_{0}(1)$, we see that we have $\xi(0, t)=\xi(1, t)$, for all $t \in[0, \infty)$. Thus the function $\xi^{*}(t)$ is a unique solution of $(8)$ with the initial condition $\xi^{*}(0)=\xi_{0}(0)$.

Note, that for $t \rightarrow \infty$

$$
\lim _{t \rightarrow \infty} \xi^{*}(t)=\left(\frac{a}{P}\right)^{\frac{1}{\gamma}}
$$

Indeed, as we may construct the appropriate Lyapunov function for the equation (8) $V(\xi) \in C^{1}(Q)$, where $Q=\left\{\xi:\left|\left(\frac{a}{P}\right)^{\frac{1}{\gamma}}-\xi\right| \leq\left|\left(\frac{a}{P}\right)^{\frac{1}{\gamma}}-\xi_{0}\right|\right\}$,

$$
V(\xi)=\left(\frac{a}{\xi^{\gamma}}-P\right)^{2} .
$$

Now, since $\nabla V \cdot \xi_{t}<0$ for $\xi \in Q \backslash\left\{\left(\frac{a}{P}\right)^{\frac{1}{\gamma}}\right\}$ we get, by the Lyapunov Stability Theorem, that $\left(\frac{a}{P}\right)^{\frac{1}{\gamma}}$ is an asymptotically stable fixed point of (8). And thus the limit in question exists. Moreover, there are exactly three possibilities for $\xi^{*}(t)$ to be bounded depending on the sign of its time derivative given by (8)

- if $\xi_{0}<\left(\frac{a}{P}\right)^{\frac{1}{\gamma}}$, then $\xi^{*}(t)$ is increasing and $0<\xi_{0} \leq \xi^{*}(t) \leq\left(\frac{a}{P}\right)^{\frac{1}{\gamma}}<\infty$, 
- if $\xi_{0}=\left(\frac{a}{P}\right)^{\frac{1}{\gamma}}$ then $\xi^{*}(t)$ is constant,

- if $\xi_{0}>\left(\frac{a}{P}\right)^{\frac{1}{\gamma}}$, then $\xi^{*}(t)$ is decreasing and $0<\left(\frac{a}{P}\right)^{\frac{1}{\gamma}} \leq \xi^{*}(t) \leq \xi_{0}<\infty$.

Define function $\theta(x, t)$ as an extension of $\xi^{*}(t)$ to the whole region $[0,1] \times[0, \infty)$ by constancy along each straight line $x=C$ :

$$
\theta(x, t)=\theta(t):=\xi^{*}(t)
$$

\section{Proof of Theorem A}

In order to prove Theorem A we first construct a finite-dimensional approximation of solutions to (1), and then pass to the limit. This is called Galerkin's method.

\subsection{Galerkin approximations}

Define the spaces:

$$
\begin{gathered}
W=\left\{\varphi \in C^{1}\left(0, T ; H^{1}(0,1)\right): \int_{0}^{1} \varphi(x) d x=0\right\}= \\
=\left\{\varphi \in C^{1}\left(0, T ; H^{1}(0,1)\right): \varphi=\sum_{k=1}^{\infty} \alpha_{k}(t) w_{k}\right\}, \\
W^{N}=\left\{\varphi \in C^{1}\left(0, T ; H^{1}(0,1)\right): \varphi=\sum_{k=1}^{N} \alpha_{k}^{N}(t) w_{k}\right\}, \\
W^{N, x}=\left\{\varphi \in C^{1}\left(0, T ; H^{1}(0,1)\right): \varphi=\sum_{k=1}^{N} \alpha_{k}^{N}(t) w_{k, x}\right\}
\end{gathered}
$$

For fixed integer $N$, we shall look for the functions $v_{N}, \xi_{N}$ of the form:

$$
v_{N}(x, t)=\theta_{t}(t)\left(x-\frac{1}{2}\right)_{N}+\sum_{k=1}^{N} \alpha_{k}^{N}(t) w_{k}(x) \quad \xi_{N}(x, t)=\theta(t) \cdot 1_{N, x}+\sum_{k=1}^{N} \beta_{k}^{N}(t) w_{k, x}
$$

where $\varphi_{N}, \varphi_{N, x}$ denote a projection of function $\varphi$ on $W^{N}, W^{N, x}$, respectively, such that for all $k=1, \ldots N$ the coefficients $\alpha_{k}^{N}(t), \beta_{k}^{N}(t)$ satisfy

$$
\alpha_{k}^{N}(0)=\left(v_{0}-\left(x-\frac{1}{2}\right)_{N} \theta_{t}(0), w_{k}\right), \quad \beta_{k}^{N}(0)=\frac{1}{(k \pi)^{2}}\left(\xi_{0}-\theta(0) \cdot 1_{N, x}, w_{k, x}\right),
$$




$$
\beta_{k}^{N}(t)=\beta_{k}^{N}(0)+\int_{0}^{t} \alpha_{k}^{N}(s) d s
$$

and

$$
\left(v_{N, t}, w_{k}\right)-\left(\frac{a}{\xi_{N}^{\gamma}}-P, w_{k, x}\right)+\mu\left(\mathbf{T} v_{N, x}, w_{k, x}\right)=0
$$

in the sense of distributions on time interval $[0, T]$.

Theorem 2 (Construction of approximate solutions). For $N$ sufficiently large there exists the unique pair of functions $v_{N}, \xi_{N}$ of the form (10) satisfying (11), (12) and (13) in the sense of distributions on time interval $[0, T]$.

For the proof see Appendix A.

In consequence, the pair of functions $v_{N}, \xi_{N}$ fulfils weak formulation in sense of the Definition for each $\varphi \in W^{N}$.

Remark 3. The assumption $\xi_{0}>0$ is equivalent to initial density $\varrho_{0}(x)=\xi_{0}^{-1}$ greater than 0 for all $x \in(0,1)$. Therefore the classical solution of second equation of (1) satisfies

$$
\xi(x, t)>0 \quad \forall(x, t) \in(0,1) \times(0, T) .
$$

Indeed, as $\xi_{t}=-\frac{\varrho t}{\varrho^{2}}$, thus $\frac{\varrho t}{\varrho}=-\varrho v_{x}$, and hence

$$
\xi^{-1}(x, t)=\varrho(x, t)=\varrho_{0}(x) \exp \left\{-\int_{0}^{t} \varrho v_{x} d t\right\}>0 .
$$

This property may be transcribed into $\xi_{N}(x, t)$ (for $N$ sufficiently large) by an analogous argument, since we observe that for $N$ sufficiently large $\xi_{N}(x, 0)>0$.

\subsection{Energy estimates}

To obtain local existence of weak solutions we need uniform estimates.

Lemma 4 (The energy equality). Let $v_{N}, \xi_{N}$ satisfy weak formulation in the sense of the Definition for each $\varphi \in W^{N}$, then

$$
\begin{aligned}
\int_{0}^{1}\left(\frac{1}{2} v_{N}^{2}(x, T)+G\left(\xi_{N}^{-1}\right)(x, T)\right) d x+P V(T) & +\mu \iint_{0}^{T}\left|\mathbf{T} v_{N, x}\right|^{2} d x d t= \\
& =\int_{0}^{1}\left(\frac{1}{2} v_{N, 0}^{2}+G\left(\xi_{N, 0}^{-1}\right)\right) d x+P V(0)
\end{aligned}
$$

holds for any $T<T_{0}$. 
Proof. Putting $\varphi=v_{N} \in W^{N}$ into the Definition, we get

$$
\left(v_{N, t}, v_{N}\right)-\left(\frac{a}{\xi_{N}^{\gamma}}-P, v_{N, x}\right)+\mu\left(\mathbf{T} v_{N, x}, v_{N, x}\right)=0
$$

Since $\mathbf{T}$ is the projector, we have

$$
\mu \int_{0}^{1} \mathbf{T} v_{N, x} v_{N, x} d x=\int_{0}^{1}\left|\mathbf{T} v_{N, x}\right|^{2} d x
$$

also by the definition of function $G(\cdot)$

$$
\begin{gathered}
-\int_{0}^{1} p\left(\xi_{N}^{-1}\right) v_{N, x} d x=-\int_{0}^{1} p\left(\xi_{N}^{-1}\right) \xi_{N, t} d x=\frac{d}{d t} \int_{0}^{1} G\left(\xi_{N}^{-1}\right) d x \\
P \int_{0}^{1} \xi_{N, t} d x=P \frac{d}{d t} \int_{0}^{1} \xi d x=\frac{d}{d t} P V(t),
\end{gathered}
$$

where $V(t)$ is the volume of fluid in the Eulerian coordinates. After these transformations we obtain an equation

$$
\frac{d}{d t} \int_{0}^{1}\left(\frac{1}{2} v_{N}^{2}+G\left(\xi_{N}^{-1}\right)\right) d x+\frac{d}{d t} P V(t)+\mu \int_{0}^{1}\left|\mathbf{T} v_{N, x}\right|^{2} d x=0
$$

integrating from 0 to $T$ we get the thesis.

Later on we will need another form of (15) which is a consequence of formula (5)

$$
\frac{d}{d t} \int_{0}^{1}\left(\frac{1}{2} v_{N}^{2}+G\left(\xi_{N}^{-1}\right)\right) d x+\frac{d}{d t} P V(t)+\mu \int_{0}^{1}\left(v_{N, x}\right)^{2} d x=-\int_{0}^{1} f_{N} v_{N} d x
$$

where we denote $f_{N}=\mu(1-\mathbf{T}) v_{N, x x}=\mu \sum_{k=1}^{R} \alpha_{k}^{N}(t) w_{k, x x}(x)$.

Remark 5. Above lemma yields an estimate on the norm of $v_{N}$ in $L_{\infty}\left(0, T ; L_{2}(0,1)\right)$, which in turn implies a suitable estimate on $\left\|f_{N}\right\|_{L_{\infty}\left(0, T ; L_{2}(0,1)\right)}$.

Lemma 6 (The energy estimate). There exists a constant $C$, depending on a, $P, \mu, \gamma$ and $T$, such that for any $T<T_{0}$ holds

$$
\sup _{t \in[0, T]}\left\|\xi_{N}(t)\right\|_{H^{1}(0,1)}+\left\|v_{N}\right\|_{L_{2}\left(0, T ; H^{1}(0,1)\right)} \leq C .
$$


Proof. This time we take as a test function $\xi_{N, x}(x, t) \in W^{N}$

$$
\left(v_{N, t}, \xi_{N, x}\right)-\left(\frac{a}{\xi_{N}^{\gamma}}-P, \xi_{N, x x}\right)+\mu\left(\mathbf{T} v_{N, x}, \xi_{N, x x}\right)=0 .
$$

Note, that transforming respective elements of this equation we get

$$
\begin{aligned}
\int_{0}^{1} v_{N, t} \xi_{N, x} d x= & \frac{d}{d t} \int_{0}^{1} v_{N} \xi_{N, x} d x-\int_{0}^{1} v_{N} \xi_{N, t x} d x=\frac{d}{d t} \int_{0}^{1} v_{N} \xi_{N, x} d x+\int_{0}^{1} v_{N, x}^{2} d x \\
-\int_{0}^{1}\left(\frac{a}{\xi_{N}^{\gamma}}-P\right) \xi_{N, x x} d x & +\mu \int_{0}^{1} \mathbf{T} v_{N, x} \xi_{N, x x} d x= \\
& =\int_{0}^{1}\left(\frac{a}{\xi_{N}^{\gamma}}\right)_{x} \xi_{N, x} d x-\mu \int_{0}^{1} \xi_{N, x t} \xi_{N, x} d x+\mu \int_{0}^{1}(1-\mathbf{T}) v_{N, x x} \xi_{N, x} d x \\
& =-\gamma \int_{0}^{1} \frac{a}{\xi_{N}^{\gamma+1}} \xi_{N, x}^{2} d x-\frac{\mu}{2} \frac{d}{d t} \int_{0}^{1} \xi_{N, x}^{2} d x+\int_{0}^{1} f_{N} \xi_{N, x} d x,
\end{aligned}
$$

altogether leads to equality

$$
\frac{d}{d t} \int_{0}^{1}\left(\frac{\mu}{2} \xi_{N, x}^{2}-v_{N} \xi_{N, x}\right) d x+\gamma a \int_{0}^{1} \frac{\xi_{N, x}^{2}}{\xi_{N}^{\gamma+1}} d x=\int_{0}^{1} v_{N, x}^{2} d x+\int_{0}^{1} f_{N} \xi_{N, x} d x .
$$

Now we multiply (16) by the constant $B=\frac{4}{\mu}$, and then add to equation (17), to find

$$
\begin{aligned}
\frac{d}{d t}\left(\int_{0}^{1}\left(\frac{\mu}{2} \xi_{N, x}^{2}+\frac{B}{2} v_{N}^{2}-v_{N} \xi_{N, x}+B G\left(\xi_{N}^{-1}\right)\right)\right. & d x+B P V(t))+ \\
& +\gamma a \int_{0}^{1} \frac{\xi_{N, x}^{2}}{\xi_{N}^{\gamma+1}} d x+(B \mu-1) \int_{0}^{1} v_{N, x}^{2} d x \\
& =\int_{0}^{1}\left(f_{N} \xi_{N, x}-B f_{N} v_{N}\right) d x .
\end{aligned}
$$

Employing to the r.h.s. of above equation Hölder's and Cauchy's inequalities and taking into account that $\frac{4}{\mu} \int_{0}^{1} G\left(\xi_{N}^{-1}\right)>0, \frac{4}{\mu} P V(t)>0$ we get

$$
\begin{gathered}
\frac{d}{d t}\left(\int_{0}^{1}\left(\frac{\mu}{2} \xi_{N, x}^{2}+\frac{2}{\mu} v_{N}^{2}-v_{N} \xi_{N, x}+\frac{4}{\mu} G\left(\xi_{N}^{-1}\right)\right) d x+\frac{4}{\mu} P V(t)\right)+\gamma a \int_{0}^{1} \frac{\xi_{N, x}^{2}}{\xi_{N}^{\gamma+1}} d x+3 \int_{0}^{1} v_{N, x}^{2} d x \\
\quad \leq \frac{5}{\mu}\left\|f_{N}\right\|_{L^{2}(0,1)}^{2}+\int_{0}^{1}\left(\frac{\mu}{2} \xi_{N, x}^{2}+\frac{2}{\mu} v_{N}^{2}-v_{N} \xi_{N, x}+\frac{4}{\mu} G\left(\xi_{N}^{-1}\right)\right) d x+\frac{4}{\mu} P V(t) .
\end{gathered}
$$


Denote:

$$
\begin{gathered}
\eta(t):=\int_{0}^{1}\left(\frac{\mu}{2} \xi_{N, x}^{2}+\frac{2}{\mu} v_{N}^{2}-v_{N} \xi_{N, x}+\frac{4}{\mu} G\left(\xi_{N}^{-1}\right)\right) d x+\frac{4}{\mu} P V(t), \\
\chi(t):=\frac{5}{\mu}\left\|f_{N}\right\|_{L^{2}(0,1)}^{2} .
\end{gathered}
$$

Using again Cauchy's inequality we deduce

$$
\eta(t) \geq \int_{0}^{1}\left(\frac{\mu}{4} \xi_{N, x}^{2}+\frac{1}{\mu} v_{N}^{2}+\frac{4}{\mu} G\left(\xi_{N}^{-1}\right)\right) d x+\frac{4}{\mu} P V(t) \geq 0 .
$$

Hence, according to our denotations, inequality (19) reads

$$
\eta^{\prime}(t) \leq \eta(t)+\chi(t)
$$

Using the Gronwall inequality we get the following estimates:

$$
\begin{aligned}
\sup _{t \in[0, T]}\left\|\xi_{N}(t)\right\|_{H^{1}(0,1)}^{2} & \leq \frac{4}{\mu} \mathrm{e}^{T}\left(\eta(0)+\frac{5}{\mu}\left\|f_{N}\right\|_{L_{2}\left(0, T ; L_{2}(0,1)\right)}^{2}\right), \\
\sup _{t \in[0, T]}\left\|v_{N}(t)\right\|_{L^{2}(0,1)}^{2} & \leq \mu \mathrm{e}^{T}\left(\eta(0)+\frac{5}{\mu}\left\|f_{N}\right\|_{L_{2}\left(0, T ; L_{2}(0,1)\right)}^{2}\right), \\
\sup _{0<t<T} V(t) & \leq \frac{\mu}{4 P} \mathrm{e}^{T}\left(\eta(0)+\frac{5}{\mu}\left\|f_{N}\right\|_{L_{2}\left(0, T ; L_{2}(0,1)\right)}^{2}\right),
\end{aligned}
$$

and

$$
\begin{aligned}
& \sup _{t \in[0, T]} \frac{4}{\mu} \int_{0}^{1} G\left(\xi_{N}^{-1}\right)(x, t) d x=\sup _{t \in[0, T]} \frac{4 a}{\mu(\gamma-1)} \int_{0}^{1} \xi_{N}^{1-\gamma}(x, t) d x= \\
& \quad=\sup _{t \in[0, T]} \frac{4 a}{\mu(\gamma-1)}\left\|\xi_{N}^{\frac{1-\gamma}{2}}(t)\right\|_{L^{2}(0,1)}^{2} \leq \mathrm{e}^{T}\left(\eta(0)+\frac{5}{\mu}\left\|f_{N}\right\|_{L_{2}\left(0, T ; L_{2}(0,1)\right)}^{2}\right) .
\end{aligned}
$$

Moreover, integrating (19) with respect to $t$ in the interval $[0, T]$ we obtain

$$
\left\|v_{N, x}\right\|_{L_{2}\left(0, T ; L_{2}(0,1)\right)}^{2}=\left\|\xi_{N, t}\right\|_{L_{2}\left(0, T ; L_{2}(0,1)\right)}^{2} \leq \frac{T \mathrm{e}^{T}+1}{2}\left(\eta(0)+\frac{5}{\mu}\left\|f_{N}\right\|_{L_{2}\left(0, T ; L_{2}(0,1)\right)}^{2}\right),
$$

and

$$
\begin{gathered}
\gamma a \int_{0}^{T} \int_{0}^{1} \frac{\left(\xi_{N, x}\right)^{2}}{\xi_{N}^{\gamma+1}}(x, t) d x d t=\frac{4 \gamma a}{(1-\gamma)^{2}} \int_{0}^{T} \int_{0}^{1}\left[\left(\xi_{N}^{\frac{1-\gamma}{2}}\right)_{x}\right]^{2}(x, t) d x d t= \\
=\frac{4 \gamma a}{(1-\gamma)^{2}}\left\|\xi_{N}^{\frac{1-\gamma}{2}}\right\|_{L_{2}\left(0, T ; H^{1}(0,1)\right)}^{2} \leq\left(T \mathrm{e}^{T}+1\right)\left(\eta(0)+\frac{5}{\mu}\left\|f_{N}\right\|_{L_{2}\left(0, T ; L_{2}(0,1)\right)}^{2}\right) .
\end{gathered}
$$


Remark 7. From the energy equality $f_{N}$ is bounded in $L_{\infty}\left(0, T ; L_{2}(0,1)\right)$, in particular

$$
\left\|f_{N}\right\|_{L_{\infty}\left(0, T ; L_{2}(0,1)\right)}^{2}<C,
$$

where $C$ is a constant, that depends on $P, a, \mu, \gamma$ and initial data, but it does not depend on $T$.

With this remark we complete the proof of the energy estimate.

Till now we have proved the following inclusions:

$$
\begin{array}{ccc}
\xi_{N} \in L_{\infty}\left(0, T ; H^{1}(0,1)\right), & \xi_{N}^{\frac{1-\gamma}{2}} \in L_{\infty}\left(0, T ; L_{2}(0,1)\right), & v_{N} \in L_{\infty}\left(0, T ; L_{2}(0,1)\right), \\
\xi_{N, t} \in L_{2}\left(0, T ; L_{2}(0,1)\right), & \xi_{N}^{\frac{1-\gamma}{2}} \in L_{2}\left(0, T ; H^{1}(0,1)\right), & v_{N} \in L_{2}\left(0, T ; H^{1}(0,1)\right) .
\end{array}
$$

At the beginning of this subsection we substantiated that for $N$ sufficiently large, $\xi_{N}(x, t)>0$, now we will prove that it is indeed separated from zero.

Lemma 8. There exists a positive constant $K$, such that for $N$ sufficiently large

$$
\xi_{N}(x, t) \geq K>0 \quad \text { for }(x, t) \in[0,1] \times[0, T] .
$$

Proof. Let $\alpha>0$, then $\sup _{t \in[0, T]} \int_{0}^{1}\left|\left(\xi_{N}^{-\alpha}\right)_{x}\right|=\sup _{t \in[0, T]} \int_{0}^{1}\left|\xi_{N}\right|^{-(\alpha+1)}\left|\xi_{N, x}\right|$. Employing the Cauchy-Schwarz inequality we obtain that for any $t \in[0, T]$

$$
\int_{0}^{1}\left|\xi_{N}\right|^{-(\alpha+1)}\left|\xi_{N, x}\right| \leq \sup _{0<t<T}\left\|\xi_{N, x}\right\|_{L^{2}(0,1)}\left(\int_{0}^{1}\left|\xi_{N}\right|^{-2(\alpha+1)}\right)^{\frac{1}{2}} .
$$

From (21) we deduce that $\xi_{N}^{-(\alpha+1)} \in L_{\infty}\left(0, T ; L_{2}(0,1)\right)$, iff $-2(\alpha+1)=1-\gamma$, and $\gamma>3$. Observe, that by the Theorem $2, \xi_{N}(x, t)$ are continuous positive functions, moreover

$$
\xi_{N}^{-\alpha} \in L_{\infty}\left(0, T ; W_{1}^{1}(0,1)\right) \subset L_{\infty}\left(0, T ; C^{0}[0,1]\right) .
$$

The norm $\left\|\xi_{N}^{-\alpha}\right\|_{L_{\infty}\left(0, T ; C^{0}[0,1]\right)} \leq M$ for some constant $M$ and $\alpha>0$, therefore we have that

$$
0<\frac{1}{M} \leq \operatorname{ess} \inf _{t \in(0, T)}\left\|\xi_{N}^{-\alpha}(t, x)\right\|_{C^{0}[0,1]}^{-1}
$$

and recalling that every $\xi_{N}(x, t)$ is positive and continuous we obtain the existence of a positive constant $K$, such that

$$
\xi_{N}(x, t) \geq K>0 .
$$

Let us underline that $K$ depends on $\mathrm{M}$ and is highly depending on $T$.

Now we shall estimate the norm of pressure

$$
\begin{aligned}
& \left\|p\left(\xi_{N}^{-1}\right)\right\|_{L_{2}\left(0, T ; H^{1}(0,1)\right)} \leq C_{P} a\left\|\left(\xi_{N}^{-\gamma}\right)_{x}\right\|_{L_{2}\left(0, T ; L_{2}(0,1)\right)} \\
& \leq C_{P} a K^{\frac{-\gamma+1}{2}}\left\|\left(\xi_{N}^{\frac{1-\gamma}{2}}\right)_{x}\right\|_{L_{2}\left(0, T ; L_{2}(0,1)\right)} \leq C_{P} a K^{\frac{-\gamma+1}{2}}\left\|\xi_{N}^{\frac{1-\gamma}{2}}\right\|_{L^{2}\left(0, T ; H^{1}(0,1)\right)}
\end{aligned}
$$

where $C_{P}$ is a constant from the Poincare inequality. According to (22) we arrive at

$$
p\left(\xi_{N}^{-1}\right) \in L_{2}\left(0, T ; H^{1}(0,1)\right) .
$$




\subsection{Existence}

The estimates from the previous subsection imply

$$
\sup _{t \in[0, T]}\left(\left\|v_{N}(t)\right\|_{L_{2}(0,1)}+\left\|\xi_{N}(t)\right\|_{H^{1}(0,1)}\right)+\left\|v_{N}\right\|_{L_{2}\left(0, T ; H^{1}(0,1)\right)}+\left\|p\left(\xi_{N}^{-1}\right)\right\|_{L_{2}\left(0, T ; H^{1}(0,1)\right)} \leq C
$$

for some constant $C$ depending on $\mu, P, \gamma, a$, initial data and $T$. As a result we may estimate $\left\|v_{N, t}\right\|_{L_{2}\left(0, T ; H^{-1}(0,1)\right)}$, where $H^{-1}(0,1)=W^{*}$.

Now we pass to limits as $N \rightarrow \infty$ to obtain a weak solution to our problem in the sense of Definition.

Theorem 9. There exists a weak solution to (1)+(2).

Proof. Since the sequence $\left\{v_{N}\right\}_{N=1}^{\infty}$ is bounded in $L_{2}\left(0, T ; H^{1}(0,1)\right)$, and $\left\{v_{N, t}\right\}_{N=1}^{\infty}$ is bounded in $L_{2}\left(0, T ; H^{-1}(0,1)\right)$, there exists a subsequence $\left\{v_{N_{l}}\right\}_{l=1}^{\infty} \subset\left\{v_{N}\right\}_{N=1}^{\infty}$, s. t.

$$
\begin{gathered}
v_{N_{l}} \rightarrow v \in L_{2}\left(0, T ; H^{1}(0,1)\right), \\
v_{N_{l}, t} \rightarrow v_{t} \in L_{2}\left(0, T ; H^{-1}(0,1)\right) .
\end{gathered}
$$

With the same manner we conclude that

$$
\begin{array}{r}
\xi_{N_{l_{k}}} \rightarrow^{*} \xi \in L_{\infty}\left(0, T ; H^{1}(0,1)\right), \\
\left(\xi_{N_{l_{k}}}-\theta \cdot 1_{N_{l_{k}}, x}\right) \rightarrow^{*} \xi^{*} \in L_{\infty}\left(0, T ; H_{0}^{1}(0,1)\right)
\end{array}
$$

for some function $\xi^{*}$ and some subsequence $\left\{\xi_{N_{l_{k}}}\right\}_{k=1}^{\infty} \subset\left\{\xi_{N_{l}}\right\}_{l=1}^{\infty}$. Starting from here we will be calling this subsequence $\xi_{N}, v_{N}$.

By virtue of $(21)$ and $(22)$ we see that $\xi_{N} \in H^{1}((0,1) \times(0, T))$, hence, by the RellichKondrachov Compactness Theorem, we obtain strong convergence of a subsequence $\left\{\xi_{N_{l}}\right\}_{l=1}^{\infty} \subset\left\{\xi_{N}\right\}_{N=1}^{\infty}$

$$
\xi_{N_{l}} \rightarrow \xi \in L_{2}((0,1) \times(0, T)) .
$$

Next, observe that since $p\left(\xi_{N}^{-1}\right)$ is bounded in $L_{2}\left(0, T ; H^{1}(0,1)\right)$ there exists a weakly convergent subsequence $\left\{\xi_{N_{l_{k}}}\right\}_{k=1}^{\infty} \subset\left\{\xi_{N_{l}}\right\}_{l=1}^{\infty}$ to some function in $L_{2}\left(0, T ; H^{1}(0,1)\right)$

$$
p\left(\xi_{N_{l_{k}}}^{-1}\right) \rightarrow \overline{p\left(\xi^{-1}\right)} \in L_{2}\left(0, T ; H^{1}(0,1)\right)
$$

Lemma 10. Providing $p\left(\xi^{-1}\right)$ is a continuous function of $\xi$ and that with an accuracy to subsequence

$$
\begin{aligned}
\xi_{N_{l}} & \rightarrow \xi \quad \text { strongly in } L_{2}((0,1) \times(0, T)), \\
p\left(\xi_{N_{l_{k}}}^{-1}\right) & \rightarrow \overline{p\left(\xi^{-1}\right)} \text { weakly in } L_{2}\left(0, T ; H^{1}(0,1)\right),
\end{aligned}
$$

then $\overline{p\left(\xi^{-1}\right)}=p\left(\xi^{-1}\right)$ holds a.e. in $(0,1) \times[0, T]$. 
The Proof of this Lemma follows easily from Egoroff's Theorem.

Similarly, since $\left\{\xi_{N}^{\frac{1-\gamma}{2}}\right\}_{N=1}^{\infty}$ is bounded in $L_{\infty}\left(0, T ; L_{2}(0,1)\right)$, we may choose such subsequence $\left\{\xi_{N_{l}}^{\frac{1-\gamma}{2}}\right\}_{l=1}^{\infty} \subset\left\{\xi_{N}^{\frac{1-\gamma}{2}}\right\}_{N=1}^{\infty}$ that

$$
\xi_{N_{l}}^{\frac{1-\gamma}{2}} \rightarrow^{*} \overline{\xi^{\frac{1-\gamma}{2}}} \in L_{\infty}\left(0, T ; L_{2}(0,1)\right)
$$

and by the same argument prove $\overline{\xi^{\frac{1-\gamma}{2}}}=\xi^{\frac{1-\gamma}{2}}$ a.e. in $(0,1) \times[0, T]$.

Repeating the procedure from proof of Lemma 8 for $\xi(x, t)$ we validate

$$
\xi(x, t) \geq C>0 \quad \text { for }(x, t) \in(0,1) \times[0, T] .
$$

Next, by the definition of the operator $\mathbf{T}$ we know that $f_{N}$ are analytic functions. Moreover the energy estimate implies that $\left\|f_{N}\right\|_{L_{2}\left(0, T ; H^{1}(0,1)\right)} \leq C(R)\left\|v_{N}\right\|_{L_{2}\left(0, T ; H^{1}(0,1)\right)} \leq C$, thus at least for some subsequence

$$
f_{N_{l}} \rightarrow^{*} \bar{f} \quad \text { weakly* in } \mathrm{L}_{\infty}\left(0, \mathrm{~T} ; \mathrm{L}_{2}(0,1)\right) .
$$

Since $(1-\mathbf{T})$ is a linear, compact operator we have that $\bar{f}=\mu[(1-\mathbf{T}) v]_{x x}=f$. According to our previous remarks, it is possible to pass to limits in the weak formulation, and by the density argument, equality

$$
\left(v_{t}, \varphi\right)-\left(p\left(\xi^{-1}\right)-P, \varphi_{x}\right)+\mu\left(v_{x}, \varphi_{x}\right)+(f, \varphi)=0
$$

holds for each $\varphi \in W$ in the sense of distributions on time interval $[0, T]$, moreover

$$
v_{x}(x, t)=\xi_{t}(x, t) \quad \text { on }(0,1) \times[0, T] .
$$

In order to prove $v(x, 0)=v_{0}(x)$, it suffices to choose for a test function such $\varphi \in W$ that $\varphi(x, T)=0$ and pass to weak limits recalling $v_{N_{l}}(x, 0) \rightarrow v_{0}(x)$ in $L_{2}(0,1)$. The equation (24) enables to show a proper initial condition for $\xi(x, t)$.

To complete the proof of existence of weak solutions there is a need to improve regularity of $v$.

\section{Lemma 11.}

$$
v \in W_{2}^{2,1}((0,1) \times(0, T)) .
$$

Proof. Multiply equation (13) by $\alpha_{k, t}^{N}$ and sum over $k=1, \ldots, N$, to find

$$
\left(v_{N, t}, \tilde{v}_{N, t}\right)-\left(p\left(\xi_{N}^{-1}\right)-P, \tilde{v}_{N, t x}\right)+\mu\left(\mathbf{T} v_{N, x}, \tilde{v}_{N, t x}\right)=0
$$

where $\tilde{v}_{N}(x, t)=\sum_{k=1}^{N} \alpha_{k}^{N}(t) w_{k}(x)$.

Let $u(x, t)=-\frac{\theta_{t}(t)}{2}+x \theta_{t}(t), u_{t}(x, t) \in W^{N}$, thus it satisfies

$$
\left(v_{N, t}, u_{t}\right)-\left(p\left(\xi_{N}^{-1}\right)-P, u_{t x}\right)+\mu\left(\mathbf{T} v_{N, x}, u_{t x}\right)=0 .
$$

Adding these two equalities and using the formula (5) we get

$$
\int_{0}^{1}\left(v_{N, t}\right)^{2} d x-\mu \int_{0}^{1} \tilde{v}_{N, x x}\left(\tilde{v}_{t}+u_{t}\right) d x=-\int_{0}^{1} p\left(\xi_{N}^{-1}\right)_{x} v_{N, t}-\mu \int_{0}^{1}(1-\mathbf{T}) v_{N, x x} v_{N, t},
$$


since $v_{N}(x, t)=u(x, t)+\tilde{v}(x, t)$, in particular $v_{N, x x}=\tilde{v}_{N, x x}$. Now employ Caychy's inequality and integrate by parts to find

$$
\begin{aligned}
\frac{\left\|v_{N, t}\right\|_{L_{2}(0,1)}^{2}}{3}+\frac{\mu}{2} \frac{d}{d t} \int_{0}^{1}\left(\tilde{v}_{N, x}\right)^{2} d x+ & \mu \int_{0}^{1} \tilde{v}_{N, x} u_{t x} \\
& \leq \frac{3}{4}\left\|p\left(\xi_{N}^{-1}\right)_{x}\right\|_{L_{2}(0,1)}^{2}+\frac{3 \mu^{2}}{4}\left\|(1-\mathbf{T}) v_{N, x x}\right\|_{L_{2}(0,1)}^{2} .
\end{aligned}
$$

Note, $\left\|\tilde{v}_{N, x}\right\|_{L_{2}\left(0, T ; L_{2}(0,1)\right)} \leq\left\|v_{N}\right\|_{L_{2}\left(0, T ; H^{1}(0,1)\right)}$ and $u_{t x}=\theta_{t t} \in L_{2}\left(0, T ; L_{2}(0,1)\right)$, therefore integrating with respect to $t$ in the interval $[0, T]$ we conclude

$$
v_{N, t} \in L_{2}\left(0, T ; L_{2}(0,1)\right) \text {. }
$$

Finally, by (25) and Cauchy-Schwarz inequality, we get that also

$$
v_{N, x x} \in L_{2}\left(0, T ; L_{2}(0,1)\right) \text {. }
$$

As $N$ approaches infinity we obtain required smoothness of $v$ and the proof of existence is complete.

\subsection{Uniqueness}

Theorem 12. The weak solution to (1)+(2) is unique.

Proof. Assume there are two weak solutions $\left(v_{1}, \xi_{1}\right)$ and $\left(v_{2}, \xi_{2}\right)$ of the system (1) in sense of the Definition.

Denote $\omega=v_{1}-v_{2}, \psi=\xi_{1}-\xi_{2}$ and subtract the weak formulations for the pairs $\left(v_{1}, \xi_{1}\right)$ and $\left(v_{2}, \xi_{2}\right)$ with $v_{1}$ and $v_{2}$ as a test functions, respectively, then we get

$$
\left(\omega_{t}, \omega\right)+\left(\left(p\left(\xi_{1}^{-1}\right)-p\left(\xi_{2}^{-1}\right)\right)_{x}, \omega\right)=\left(\mu(\mathbf{T} \omega)_{x x}, \omega\right) .
$$

Employing formula $h\left(\xi_{1}\right)-h\left(\xi_{2}\right)=\left(\xi_{1}-\xi_{2}\right) \int_{0}^{1} h^{\prime}\left(s \xi_{1}+(1-s) \xi_{2}\right) d s$, for $h(\xi)=p\left(\xi^{-1}\right)$, integrating by parts and replacing $\omega_{x}$ by $\psi_{t}$, we arrive at

$$
\begin{aligned}
\frac{d}{d t} \int_{0}^{1}\left(\frac{\omega^{2}}{2}-\frac{\psi^{2}}{2} \int_{0}^{1} h^{\prime}\left(s \xi_{1}+(1-s) \xi_{2}\right) d s\right) d x= \\
\quad=-\int_{0}^{1} \frac{\psi^{2}}{2} \int_{0}^{1} h^{\prime \prime}\left(s \xi_{1}+(1-s) \xi_{2}\right) d s \xi_{t} d x-\mu \int_{0}^{1}\left(\mathbf{T} \omega_{x}\right)^{2} d x \\
\leq C(t)\left\|\xi_{t}(t)\right\|_{L_{\infty}(0,1)} \int_{0}^{1} \frac{\psi^{2}}{2} d x
\end{aligned}
$$


where $C(t)=\left\|\int_{0}^{1} h^{\prime \prime}\left(s \xi_{1}+(1-s) \xi_{2}\right) d s(t)\right\|_{L_{\infty}(0,1)}, \sup _{t \in[0, T]} C(t)<\infty$. Due to result from previous subsection $\xi_{t} \in L_{2}\left(0, T ; H^{1}(0,1)\right) \subset L_{1}\left(0, T ; L_{\infty}(0,1)\right)$.

Since $h(\xi)=\frac{a}{\xi^{\gamma}}$ is monotonically decreasing function, its derivative is negative, thus

$$
\frac{\omega^{2}}{2}-\frac{\psi^{2}}{2} \int_{0}^{1} h^{\prime}\left(s \xi_{1}+(1-s) \xi_{2}\right) d s \geq 0 .
$$

Let $\phi(t)=C(t)\left\|\xi_{t}(t)\right\|_{L_{\infty}(0,1)}$, note $\phi \in L_{1}(0, T)$, then by Gronwall's inequality and the initial conditions $\omega(x, 0)=0, \psi(x, 0)=0$

$$
\int_{0}^{1}\left(\frac{\omega^{2}}{2}-\frac{\psi^{2}}{2} \int_{0}^{1} h^{\prime}\left(s \xi_{1}+(1-s) \xi_{2}\right) d s\right) d x \leq 0,
$$

therefore $\psi(x, t)=\omega(x, t) \equiv 0$ a.e.

\section{Proof of Theorem B}

To obtain a global in time existence in case when the local existence has been already proved we only have to show uniform in time estimates for solutions of (1).

Lemma 13. For a solution of $(1)+(2)$ we have

$$
\begin{aligned}
\int_{0}^{1}\left(\frac{1}{2} v^{2}(x, t)+G\left(\xi^{-1}\right)(x, t)\right) d x+P V(t)+\mu & \int_{0}^{t} \int_{0}^{1}\left|\mathbf{T} v_{x}\right|^{2} d x d s= \\
& =\int_{0}^{1}\left(\frac{1}{2} v_{0}^{2}+G\left(\xi_{0}^{-1}\right)\right) d x+P V(0) .
\end{aligned}
$$

Proof. Multiplying the first equation of (1) by $v$, integrating over [0, 1] and repeating the proof of Lemma 4 we arrive at

$$
\frac{d}{d t} \int_{0}^{1}\left(\frac{1}{2} v^{2}+G\left(\xi^{-1}\right)\right) d x+\frac{d}{d t} P V(t)+\mu \int_{0}^{1}\left|\mathbf{T} v_{x}\right|^{2} d x=0
$$

integrating over $[0, t]$ we get thesis.

As a result:

$$
\begin{aligned}
v & \in L_{\infty}\left(0, \infty ; L_{2}(0,1)\right), & & \mathbf{T} v_{x} \in L_{2}\left(0, \infty ; L_{2}(0,1)\right), \\
G\left(\xi^{-1}\right) & \in L_{\infty}\left(0, \infty ; L_{1}(0,1)\right), & & V(t) \in L_{\infty}(0, \infty) .
\end{aligned}
$$

Now we will show that for $\xi$ holds $\xi \in L_{\infty}\left(0, \infty ; H^{1}(0,1)\right)$. 
Lemma 14 (The upper bound on $\xi$ ). If $\frac{P}{\mu}$ is sufficiently large, then

$$
\xi(x, t) \leq \max \left\{\xi_{\text {min }}, \sup _{x \in(0,1)}\left(\xi_{0}+\frac{1}{\mu} U(x, 0)\right)+\frac{1}{\mu} M_{U}-t \frac{P}{4 \mu}\right\}
$$

for $t \in\left[0, T_{\max }\right)$ and a constant $\xi_{\min }$, where $T_{\max }$ is the maximal time of existence of solution in sense of the Definition.

Proof. Define a function $U(x, t)=\int_{0}^{x} v(s, t) d s$. Since $\int_{0}^{1} v(x, t) d x=0$, we have $U(0, t)=U(1, t)=0$, and consequently

$$
\left(v_{t}, \varphi\right)=\left(U_{x t}, \varphi\right)=-\left(U_{t}, \varphi_{x}\right)
$$

Therefore we may rewrite the weak formulation in the form

$$
\left(\left(\xi-\frac{1}{\mu} U\right)_{t}, \varphi_{x}\right)=\left(\frac{1}{\mu}\left(p\left(\xi^{-1}\right)-P\right)+(1-\mathbf{T}) \xi_{t}, \varphi_{x}\right) .
$$

Note, that by $(27), U \in L_{\infty}\left(0, \infty ; H_{0}^{1}(0,1)\right) \subset L_{\infty}((0, \infty) \times(0,1))$, moreover by properties of the operator $\mathbf{T}$, we see that $(1-\mathbf{T}) v_{x} \in L_{\infty}((0, \infty) \times(0,1))$. In particular, the following bounds are true

$$
\|U\|_{L_{\infty}((0, \infty) \times(0,1))} \leq M_{U}, \quad\left\|(1-\mathbf{T}) \xi_{t}\right\|_{L_{\infty}((0, \infty) \times(0,1))} \leq C .
$$

The constant $\mathrm{C}$ is independent of $\mu$, thus we choose $\mu$ sufficiently small to keep

$$
\frac{-P}{\mu}+(1-\mathbf{T}) \xi_{t} \leq \frac{-P}{2 \mu} .
$$

Let $F(\xi)=\frac{a}{\xi^{\gamma}}$ and $\xi_{\text {min }}$ be a minimal positive constant which satisfies $F\left(\xi_{\text {min }}\right)<\frac{P}{4}$, since $F(\xi)$ is a continuous decreasing function it can be done for any case, and for such $\xi_{\text {min }}$ holds

$$
\frac{1}{\mu} p\left(\xi_{\text {min }}^{-1}\right)-\frac{P}{\mu}+(1-\mathbf{T}) \xi_{t} \leq \frac{-P}{4 \mu} .
$$

Next, define a set $N=\left\{(x, t): \xi(x, t)-\frac{1}{\mu} U(x, t) \geq \xi_{\text {min }}-\frac{1}{\mu} M_{U}\right\}$.

By this definition, remembering that $F(\xi)$ is decreasing, we see that

$$
\left.\xi\right|_{N} \geq \xi_{\text {min }}
$$

Since we require only that $\varphi \in W$, i.e. $\int_{0}^{1} \varphi(x, t)=0$, hence there is no restriction on $\varphi_{x}$ which appears in (29). Choosing $\varphi$ such that $\operatorname{supp} \varphi_{x} \subset N$, we obtain a point wise inequality:

$$
\left.\frac{\partial}{\partial t}\left(\xi-\frac{1}{\mu} U\right)\right|_{N} \leq \frac{-P}{4 \mu}
$$

Then there are two possibilities:

1. If $\sup _{x \in(0,1)}\left(\xi_{0}(x)-\frac{1}{\mu} U(x, 0)\right) \leq \xi_{\text {min }}-\frac{1}{\mu} M_{U}$, then if there was

$$
\sup _{(x, t) \in(0,1) \times\left(0, T_{\max }\right)}\left(\xi(x, t)-\frac{1}{\mu} U(x, t)\right)>\xi_{\min }-\frac{1}{\mu} M_{U},
$$


it would exist a point $\left(x_{0}, t_{0}\right)$ s.t. $\xi\left(x_{0}, t_{0}\right)-\frac{1}{\mu} U\left(x_{0}, t_{0}\right)=\xi_{\min }-\frac{1}{\mu} M_{U}$, since the function $\xi(x, t)-\frac{1}{\mu} U(x, t)$ is continuous for $(x, t) \in(0,1) \times\left(0, T_{\max }\right)$. But, by virtue of $(30)$ the function $\xi(x, t)-\frac{1}{\mu} U(x, t)$ is monotonically decreasing in $\left(x_{0}, t_{0}\right)$

$$
\frac{\partial}{\partial t}\left(\xi\left(x_{0}, t_{0}\right)-\frac{1}{\mu} U\left(x_{0}, t_{0}\right)\right) \leq \frac{-P}{4 \mu},
$$

and this is a contradiction to (31). Thus, the following condition is fulfilled

$$
\sup _{(x, t) \in(0,1) \times\left(0, T_{\max }\right)}\left(\xi(x, t)-\frac{1}{\mu} U(x, t)\right) \leq \xi_{\min }-\frac{1}{\mu} M_{U}
$$

and since $U(x, t) \in L_{\infty}((0,1) \times(0, \infty))$ the function $\xi(x, t)$ is bounded for all $(x, t) \in$ $(0,1) \times(0, \infty)$.

2. If $\sup _{x \in(0,1)}\left(\xi_{0}(x)-\frac{1}{\mu} U(x, 0)\right)>\xi_{\text {min }}-\frac{1}{\mu} M_{U}$, then, by the continuity and monotonicity of function $\xi(x, t)-\frac{1}{\mu} U(x, t)$ for $(x, t) \in N$ (see the condition (30)) there exists $T_{\min }$ s. t. for $t \in\left[0, T_{\text {min }}\right]$ the function $\xi(x, t)-\frac{1}{\mu} U(x, t)$ decreases monotically until it reaches the value $\xi_{\text {min }}-\frac{1}{\mu} M_{U}$. We may compute $T_{\text {min }}$ from the condition (30)

$$
T_{\min }=\frac{4 \mu}{P}\left(\sup _{x \in(0,1)}\left(\xi_{0}-\frac{1}{\mu} U(x, 0)\right)-\xi_{\min }+\frac{1}{\mu} M_{U}\right) .
$$

Thus for $t \in\left[0, T_{\min }\right]$ we have

$$
\xi(x, t) \leq \sup _{x \in(0,1)}\left(\xi_{0}-\frac{1}{\mu} U(x, 0)\right)+\frac{1}{\mu} M_{U}-t \frac{P}{4 \mu} .
$$

and for $t \in\left(T_{\min }, \infty\right)$ the bound from previous case remains valid.

Combining these two case we complete the proof.

Recapitulating, here exists a constant $\xi_{+}$s. t. $\xi(x, t) \leq \xi_{+}$for all $(x, t) \in(0,1) \times[0, \infty)$.

Lemma 15. For $\mu \leq \frac{a \gamma}{\xi_{+}^{\gamma+1}}$ there exists a constant $K$ depending on $P, a, \gamma$ and the initial data, such that

$$
\sup _{t \in[0, \infty)}\|\xi\|_{H^{1}(0,1)} \leq K
$$

Proof. Multiplying the first equation of (1) by $\xi_{x}$, integrating over $x \in[0,1]$ and rearranging it the same way we did proving Lemma 6 , we find

$$
\frac{d}{d t} \int_{0}^{1}\left(\frac{\mu}{2} \xi_{x}^{2}-v \xi_{x}\right) d x+\gamma a \int_{0}^{1} \frac{\xi_{x}^{2}}{\xi^{\gamma+1}} d x=\int_{0}^{1} v_{x}^{2} d x+\int_{0}^{1} f \xi_{x} d x-\theta_{t} \int_{0}^{1} v_{x} d x
$$

where $f(x, t)=\mu(1-\mathbf{T}) v_{x x} \in L_{\infty}\left(0, \infty ; L_{2}(0,1)\right)$ and the presence of the last term is a result of integrating $v_{t} \xi_{x}$ over $x \in[0,1]$ by parts. 
Now we multiply equation (26) by $\frac{4}{\mu}$ and add to (32), then recalling the formula $\mu \int_{0}^{1}\left|T v_{x}\right|^{2} d x=\mu \int_{0}^{1}\left|v_{x}\right|^{2} d x+\int_{0}^{1} f v d x$, we get

$$
\begin{aligned}
\frac{d}{d t}\left(\int_{0}^{1}\left(\frac{\mu}{2} \xi_{x}^{2}+\frac{2}{\mu} v^{2}-v \xi_{x}+\frac{4}{\mu} G\left(\xi^{-1}\right)\right) d x+\right. & \left.\frac{4}{\mu} P V(t)\right)+\gamma a \int_{0}^{1} \frac{\xi_{x}^{2}}{\xi^{\gamma+1}} d x+3 \int_{0}^{1} v_{x}^{2} d x= \\
& =\int_{0}^{1}\left(f \xi_{x}-\frac{4}{\mu} f v\right) d x-\theta_{t} \int_{0}^{1} v_{x} d x .
\end{aligned}
$$

It follows from the previous lemma that

$$
\int_{0}^{1} \frac{\left(\xi_{x}\right)^{2}}{\xi^{\gamma+1}} d x \geq \frac{1}{\xi_{+}^{\gamma+1}} \int_{0}^{1} \xi_{x}^{2}
$$

Since $\mu<\frac{\gamma a}{\xi_{+}^{\gamma+1}}$ by the Poincare's (with a constant $C_{P}$ ) and Cauchy's $\left(a b \leq \epsilon a^{2}+\frac{b^{2}}{4 \epsilon}\right)$ inequalities we obtain

$$
\begin{gathered}
\frac{d}{d t}\left(\int_{0}^{1}\left(\frac{\mu}{2} \xi_{x}^{2}+\frac{2}{\mu} v^{2}-v \xi_{x}+\frac{4}{\mu} G\left(\xi^{-1}\right)\right) d x+\frac{4}{\mu} P V(t)\right)+\int_{0}^{1}\left(\frac{\mu}{2} \xi_{x}^{2}+\frac{2}{\mu} v^{2}-v \xi_{x}\right) d x \\
\quad \leq \int_{0}^{1}\left(f \xi_{x}-\frac{4}{\mu} f v\right) d x+\frac{1}{4}\left|\theta_{t}\right|^{2}-\frac{2}{C_{P}^{2}} \int_{0}^{1} v^{2} d x+\int_{0}^{1}\left(-\frac{\mu}{2} \xi_{x}^{2}+\frac{2}{\mu} v^{2} d x-v \xi_{x}\right) d x .
\end{gathered}
$$

Employing again the Cauchy's inequality we can transform the right hand side of the above expression into

$$
\begin{array}{r}
\frac{d}{d t}\left(\int_{0}^{1}\left(\frac{\mu}{2} \xi_{x}^{2}+\frac{2}{\mu} v^{2}-v \xi_{x}+\frac{4}{\mu} G\left(\xi^{-1}\right)\right) d x+\frac{4}{\mu} P V(t)\right)+\int_{0}^{1}\left(\frac{\mu}{2} \xi_{x}^{2}+\frac{2}{\mu} v^{2}-v \xi_{x}\right) d x \\
\leq \frac{5}{\mu}\|f\|_{L_{2}(0,1)}^{2}+\frac{1}{4}\left|\theta_{t}\right|^{2}+\left(\frac{4}{\mu}-\frac{2}{C_{P}^{2}}\right) \int_{0}^{1} v^{2} d x
\end{array}
$$

Denoting:

$$
\eta(t)=\int_{0}^{1}\left(\frac{\mu}{2} \xi_{x}^{2}+\frac{2}{\mu} v^{2}-v \xi_{x}+\frac{4}{\mu} G\left(\xi^{-1}\right)\right) d x+\frac{4}{\mu} P V(t),
$$

the inequality (34) reads

$$
\frac{d}{d t} \eta(t)+\eta(t) \leq \frac{5}{\mu}\|f\|_{L_{2}(0,1)}^{2}+\frac{1}{4}\left|\theta_{t}\right|^{2}+\left(\frac{4}{\mu}-\frac{2}{C_{P}}\right) \int_{0}^{1} v^{2} d x+\int_{0}^{1} \frac{4}{\mu} G\left(\xi^{-1}\right) d x+\frac{4}{\mu} P V(t) .
$$


By virtue of $(27),(28)$, the r.h.s. of this inequality is bounded in $L_{\infty}(0, \infty)$ by some constant $M_{\eta}$. Due to the Gronwall inequality the following estimate holds

$$
\eta(t) \leq \eta(0) \mathrm{e}^{-t}+M_{\eta} \mathrm{e}^{-t}+M_{\eta}
$$

thus

$$
\sup _{t \in[0, \infty)} \eta(t) \leq \eta(0)+2 M_{\eta}
$$

Finally, by Cauchy's inequality $\eta(t) \geq \int_{0}^{1}\left(\frac{\mu}{4} \xi_{x}^{2}+\frac{1}{\mu} v^{2}+\frac{4}{\mu} G\left(\xi^{-1}\right)\right) d x+\frac{4}{\mu} P V(t)$, therefore we truly have $\xi \in L_{\infty}\left(0, \infty ; H^{1}(0,1)\right)$.

Remark 16. The inclusion (28) is equivalent to $\xi^{\frac{1-\gamma}{2}} \in L_{\infty}\left(0, \infty ; L_{2}(0,1)\right)$. Since $\xi \in L_{\infty}\left(0, \infty ; H^{1}(0,1)\right)$, we may repeat an argument from Lemma 8 to obtain the existence of a constant $\xi_{-}>0$ s. $t . \xi(x, t) \geq \xi_{-}>0$ for all $(x, t) \in(0,1) \times[0, \infty)$.

By the Theorem A solution may be extended to the whole half line $t \in[0, \infty)$, with this observation the proof of global in time existence is complete.

\section{A Proof of Theorem 2}

Since $\left(w_{k}, w_{j}\right)=\delta_{k j}$, thus for $k=1, \ldots, N$ we have

$$
\begin{gathered}
\left(v_{N, t}, w_{k}\right)=\alpha_{k, t}^{N}(t)+\theta_{t}(t)\left(\left(x-\frac{1}{2}\right)_{N}, w_{k}\right), \\
\left(\frac{a}{\xi_{N}^{\gamma}}-P, w_{k, x}\right)=\left(\frac{a}{\left(\theta(t) \cdot 1_{N, x}+\sum_{k=1}^{N}\left[\beta_{k}^{N}(0)+\int_{0}^{t} \alpha_{k}^{N}(s) d s\right] w_{k, x}\right)^{\gamma}}-P, w_{k, x}\right),
\end{gathered}
$$

where $\theta(t)$ is a differentiable function defined in the Section $2, \beta_{k}^{N}(0)=\left(\xi_{0}-\pi(0), w_{k, x}\right)$ and

$$
\left(\mathbf{T} v_{N, x}, w_{k, x}\right)=\left\{\begin{array}{ll}
\left(\theta_{t}(t)\left(1_{N, x}, w_{k, x}\right)+\alpha_{k}^{N}\left(w_{k, x}, w_{k, x}\right)\right. & \text { if } k \geq R+1 \\
0 & \text { if } k<R+1
\end{array} .\right.
$$

Thus, to prove the theorem we should show that there exists unique solution to a system of ODE

$$
\begin{aligned}
\alpha_{t}^{N}(t) & =f^{N}\left(\alpha^{N}, t\right), \\
\alpha^{N}(0) & =\alpha_{0}^{N},
\end{aligned}
$$

for the time interval $t \in\left[0, T_{0}\right)$, where

$$
\begin{gathered}
\alpha^{N}(t)=\left(\alpha_{1}^{N}(t), \ldots, \alpha_{N}^{N}(t)\right) \\
f^{N}\left(\alpha^{N}, t\right): \mathbb{R}^{N+1} \rightarrow \mathbb{R}^{N}, \quad f^{N}=\left(f_{1}^{N}\left(\alpha^{N}, t\right), \ldots, f_{N}^{N}\left(\alpha^{N}, t\right)\right)
\end{gathered}
$$


and

$$
\begin{aligned}
& f_{k}^{N}\left(\alpha^{N}, t\right)=\left(\frac{a}{\left(\theta(t) \cdot 1_{N, x}+\sum_{k=1}^{N}\left[\beta_{k}^{N}(0)+\int_{0}^{t} \alpha_{k}^{N}(s) d s\right] w_{k, x}\right)^{\gamma}}-P, w_{k, x}\right) \\
& -\theta_{t}(t)\left(\left(x-\frac{1}{2}\right)_{N}, w_{k}\right)-\left\{\begin{array}{ll}
\left(\theta_{t}(t)\left(1_{N, x}, w_{k, x}\right)+\alpha_{k}^{N}\left(w_{k, x}, w_{k, x}\right)\right. & \text { if } k \geq R+1 \\
0 & \text { if } k<R+1
\end{array} .\right.
\end{aligned}
$$

Note, that since the initial value belongs to $H^{1}(0,1) \subset C[0,1]$ and $\xi(x, 0) \geq \xi_{0-}>0$, we have that for sufficiently large $N$ the orthogonal projection of the function $\xi(x, 0)$ on a subspace spanned by the first $N$ vectors from the base $\left\{w_{k, x}\right\}_{k=1}^{\infty}$ is also separated from 0, i.e. $\xi_{N}(x, 0) \geq \xi_{0-}-\epsilon>0$, where $\epsilon \rightarrow 0$ for $N \rightarrow \infty$.

From the energy equality we have the a priori bound $\left|\alpha^{N}(t)\right|<C(T)$.

Now, by the continuity of $\int_{0}^{t} \alpha_{k}^{N}(s) d s$ and by the continuity and positiveness of function $\theta(x, t)=\theta(t)$ we deduce that there exists $T>\vartheta(N)>0$ such that $\xi_{N}(x, t) \geq \frac{\xi_{0-}}{2}>0$ for $t \in[0, \vartheta]$.

Consider a set

$$
Q=\left\{\left(t, \alpha^{N}\right): t \in[0, \vartheta], \quad\left|\alpha^{N}-\alpha_{0}^{N}\right| \leq \delta\right\}
$$

on which $f^{N}\left(\alpha^{N}, t\right)$ is a continuous function, thus achieves its bounds, assume

$$
\sup _{\left(t, \alpha^{N}\right) \in Q}\left|f^{N}\left(\alpha^{N}, t\right)\right|=M
$$

Employing Banach Fixed Point Theorem we will show that the following map

$$
F\left(\alpha^{N}\right)(t)=\alpha_{0}^{N}+\int_{0}^{t} f^{N}\left(s, \alpha^{N}(s)\right) d s
$$

has exactly one fixed point, which is indeed a local solution to the system (35).

Establish $k \geq R+1$, and take $\alpha^{N, 1}, \alpha^{N, 2} \in S$, where

$$
S=\left\{\alpha^{N}(t): \alpha^{N}(0)=\alpha_{0}^{N}, t \in[0, \tau],\left|\alpha^{N}-\alpha_{0}^{N}\right| \leq \delta\right\}
$$

is a closed subset of metric space of continuous functions, thus complete metric space, with $\tau<\min \left\{\vartheta, \frac{\delta}{M}\right\}$.

Denote

$$
\xi_{N, i}(x, t)=\theta(t) \cdot 1_{N, x}+\sum_{k=1}^{N}\left[\beta_{k}^{N}(0)+\int_{0}^{t} \alpha_{k}^{N, i}(s) d s\right] w_{k, x}, \quad i=1,2,
$$


then the following sequence of inequalities is valid

$$
\begin{aligned}
\mid f_{k}^{N}\left(\alpha^{N, 1}(t), t\right) & -f_{k}^{N}\left(\alpha^{N, 2}(t), t\right) \mid \\
& \leq a \int_{0}^{1}\left|\frac{w_{k, x}\left(\xi_{N, 2}^{\gamma}(x, t)-\xi_{N, 1}^{\gamma}(x, t)\right)}{\xi_{N, 1}^{\gamma}(x, t) \xi_{N, 2}^{\gamma}(x, t)}\right| d x+\left|\alpha_{k}^{N, 1}(t)-\alpha_{k}^{N, 2}(t)\right| \int_{0}^{1} w_{k, x}^{2} d x \\
& \leq \frac{a}{\left(\frac{\xi_{0-}}{2}\right)^{2 \gamma}} \int_{0}^{1}\left|w_{k, x}\left(\xi_{N, 2}^{\gamma}(x, t)-\xi_{N, 1}^{\gamma}(x, t)\right)\right| d x+\left|\alpha_{k}^{N, 1}(t)-\alpha_{k}^{N, 2}(t)\right| \int_{0}^{1} w_{k, x}^{2} d x \\
& \leq \frac{2^{2 \gamma} a k \pi}{\xi_{0-}^{2 \gamma}\|\cos (\pi k x)\|_{L_{2}(0,1)}} L_{\gamma} \int_{0}^{1}\left|\xi_{N, 2}(x, t)-\xi_{N, 1}(x, t)\right| d x+k^{2} \pi^{2}\left|\alpha_{k}^{N, 1}(t)-\alpha_{k}^{N, 2}(t)\right|,
\end{aligned}
$$

where $L_{\gamma}$ is a Lipschitz constant for a function $f(\xi)=\xi^{\gamma}$ on $S$.

$$
\begin{aligned}
\int_{0}^{1}\left|\xi_{N, 2}(x, t)-\xi_{N, 1}(x, t)\right| d x & =\int_{0}^{1}\left|\sum_{k=1}^{N}\left(\int_{0}^{t} \alpha_{k}^{N, 2}(s) d s-\int_{0}^{t} \alpha_{k}^{N, 1}(s) d s\right) w_{k, x}\right| d x \\
& \leq \frac{k \pi}{\|\cos (k \pi x)\|_{L_{2}(0,1)}} \sum_{k=1}^{N} \int_{0}^{t}\left|\alpha_{k}^{N, 2}(s)-\alpha_{k}^{N, 1}(s)\right| d s \\
& \leq \frac{k \pi}{\|\cos (k \pi x)\|_{L_{2}(0,1)}} \sum_{k=1}^{N} \tau \sup _{t \in[0, \tau]}\left|\alpha_{k}^{N, 2}(t)-\alpha_{k}^{N, 1}(t)\right|
\end{aligned}
$$

$F^{N}$ maps $S$ into itself because

$$
\begin{aligned}
\sup _{t \in[0, \tau]}\left|F^{N}\left(\alpha^{N}\right)(t)-\alpha_{0}^{N}\right| \leq \sup _{t \in[0, \tau]} \int_{0}^{t}\left|f^{N}\left(\alpha^{N}(s), s\right)\right| d s \\
\quad \leq \sup _{t \in[0, \tau]} \int_{0}^{t} \sup _{s \in[0, t]}\left|f^{N}\left(\alpha^{N}(s), s\right)\right| d s \leq M \tau \leq \delta .
\end{aligned}
$$


One more thing needed to show is that $F^{N}$ is a contraction

$$
\begin{aligned}
\sup _{t \in[0, \tau]}\left|F^{N}\left(\alpha^{N, 1}\right)(t)-F^{N}\left(\alpha^{N, 2}\right)(t)\right| & \leq \sup _{t \in[0, \tau]} \int_{0}^{t}\left|f^{N}\left(\alpha^{N, 1}\right)(s)-f^{N}\left(\alpha^{N, 2}\right)(s)\right| d s \\
& \leq \sum_{k=1}^{N} \sup _{t \in[0, \tau]} \int_{0}^{t}\left|f_{k}^{N}\left(\alpha^{N, 1}\right)(s)-f_{k}^{N}\left(\alpha^{N, 2}\right)(s)\right| d s \\
& \leq \sum_{k=1}^{N} \sup _{t \in[0, \tau]} \int_{0}^{t} \sup _{s \in[0, t]}\left|f_{k}^{N}\left(\alpha^{N, 1}\right)(s)-f_{k}^{N}\left(\alpha^{N, 2}\right)(s)\right| d s \\
& \leq \tau \sum_{k=1}^{N} \sup _{t \in[0, \tau]}\left|f_{k}^{N}\left(\alpha^{N, 1}\right)(t)-f_{k}^{N}\left(\alpha^{N, 2}\right)(t)\right|,
\end{aligned}
$$

from the previous calculations it follows that

$$
\begin{aligned}
& \sup _{t \in[0, \tau]}\left|f_{k}^{N}\left(\alpha^{N, 1}\right)(t)-f_{k}^{N}\left(\alpha^{N, 2}\right)(t)\right| \\
\leq & \frac{2^{2 \gamma} a k^{2} \pi^{2}}{\xi_{0-}^{2 \gamma}\|\cos (k \pi x)\|_{L_{2}(0,1)}^{2}} L_{\gamma} \sum_{k=1}^{N} \tau \sup _{t \in[0, \tau]}\left|\alpha_{k}^{N, 2}(t)-\alpha_{k}^{N, 1}(t)\right|+\sup _{t \in[0, \tau]} k^{2} \pi^{2}\left|\alpha_{k}^{N, 1}(t)-\alpha_{k}^{N, 2}(t)\right|,
\end{aligned}
$$

summing over $k=1, \ldots, N$ we arrive at

$$
\begin{aligned}
\sup _{t \in[0, \tau]} \mid F^{N}\left(\alpha^{N, 1}\right)(t)- & F^{N}\left(\alpha^{N, 2}\right)(t) \mid \\
\leq & \tau \sum_{k=1}^{N}\left(\frac{a k^{2} \pi^{2} N}{C^{2 \gamma}\|\cos (k \pi x)\|_{L_{2}(0,1)}^{2}} L_{\gamma}\right)\left(\sum_{k=1}^{N} \tau \sup _{t \in[0, \tau]}\left|\alpha_{k}^{N, 2}(t)-\alpha_{k}^{N, 1}(t)\right|\right) \\
& +\sup _{t \in[0, \tau]} \tau \sum_{k=1}^{N} k^{2} \pi^{2}\left|\alpha_{k}^{N, 1}(t)-\alpha_{k}^{N, 2}(t)\right| \\
\leq & Z \sup _{t \in[0, \tau]} \sum_{k=1}^{N}\left|\alpha_{k}^{N, 1}(t)-\alpha_{k}^{N, 2}(t)\right| \leq Z N \sup _{t \in[0, \tau]}\left|\alpha^{N, 1}(t)-\alpha^{N, 2}(t)\right| .
\end{aligned}
$$

Now we take sufficiently small $\tau$ in order to have $Z N<1$ and referring to the Banach Theorem we get existence and uniqueness of solution to the system (35) on the time interval $t \in[0, \tau]$.

Recalling that $\xi_{N}(x, t)$ is separated from zero and $\alpha^{N}(t)$ is bounded by a constant for all $t<T_{0}$, we may extend the local solution to the whole interval $\left[0, T_{0}\right)$.

\section{ACKNOWLEDGEMENTS}

The author wishes to express her gratitude to Piotr Bogusław Mucha for suggesting the problem, stimulating conversations and help during preparation of the paper. 
The author was supported by the International Ph.D. Projects Programme of Foundation for Polish Science operated within the Innovative Economy Operational Programme 2007-2013 funded by European Regional Development Fund (Ph.D. Programme: Mathematical Methods in Natural Sciences) and partly supported by the MN Grant No N N201 547438.

\section{References}

[1] Y. Qin, L. Huang, Z. Yao: A remark on regularity of 1D compressible isentropic Navier-Stokes equations with density-dependent viscosity, J. Math. Anal. Appl. 351 (2009) 497-508

[2] P. B. Mucha: Private Communication, 2008

[3] T. Zhang, D.Fang: Global Behavior of Compressible Navier-Stokes Equations with Degenerate Viscosity Coefficient, Arch. Rational Mech. Anal. 182 (2006) 223-253

[4] B. Ducomet, A. Zlotnik: Lyapunov Functional Method for 1D Radiative and Reactive Viscous Gas Dynamics, Arch. Ration. Mech. Anal. 177, 185-229 (2005)

[5] B. Ducomet, E. Feireisl: On the Dynamics of Gaseous Stars, Arch. Ration. Mech. Anal. 174, 221-266 (2004)

[6] M. Lewicka, P. B. Mucha: On temporal asymptotics for the p'th power viscous reactive gas, Nonlinear Anal. 57 (2004), no. 7-8, 951-969.

[7] A. Novotný, I. Straškraba: Introduction to the Mathematical Theory of Compressible Flow, Oxford University Press (2004)

[8] P. B. Mucha: Compressible Navier-Stokes system in 1-D, Math. Meth. Appl. Sci. 2001; 24:607-622

[9] P.-L. Lions, B. Perthame, P.E. Souganidis: Existence and stability of entropy solutions for the hyperbolic systems of isentropic gas dynamics in Eulerian and Lagrangian coordinates. Comm. Pure Appl. Math. 49 (1996), no. 6, 599-638.

[10] A. Matsumura, S. Yanagi: Uniform Boundedness of the Solutions for a OneDimensional Isentropic Model System of Compressible Viscous Gas, Comm. Math. Phys. 175, 259-274 (1996)

[11] P.-L. Lions, B. Perthame, E. Tadmor: Kinetic formulation of the isentropic gas dynamics and p-systems. Comm. Math. Phys. 163 (1994), no. 2, 415-431.

[12] W.M. Zajączkowski: On nonstationary motion of a compressible barotropic viscous fluid bounded by a free surface, Dissertationes Math. 1993; 324

[13] S.N. Shore: An introduction to astrophysical hydrodynamics, New York: Academic Press, 1992 
[14] A.V. Kazhikhov: Correctness in the large of initial-boundary value problem for model system of equations of a viscous gas, Din. Sploshnoi Sredy 1975; 21:18-47 